\title{
Gauge/Gravity Dynamics for Composite Higgs Models and the Top Mass
}

\author{
Johanna Erdmenger, ${ }^{1, *}$ Nick Evans, ${ }^{2, \dagger}$ Werner Porod®, ${ }^{1, \$}$ and Konstantinos S. Rigatos $\circledast^{2, \S}$ \\ ${ }^{1}$ Institute for Theoretical Physics and Astrophysics, Julius-Maximilians-Universität Würzburg, 97074 Würzburg, Germany \\ ${ }^{2}$ School of Physics \& Astronomy and STAG Research Centre, University of Southampton, \\ Highfield, Southampton SO17 1BJ, United Kingdom
}

(Received 8 October 2020; accepted 22 January 2021; published 19 February 2021)

\begin{abstract}
We provide gauge/gravity dual descriptions of explicit realizations of the strong coupling sector of composite Higgs models using insights from nonconformal examples of the AdS/CFT correspondence. We calculate particle masses and pion decay constants for proposed $\mathrm{Sp}(4)$ and $\mathrm{SU}(4)$ gauge theories, where there is the best lattice data for comparison. Our results compare favorably to lattice studies and go beyond those due to a greater flexibility in choosing the fermion content. That content changes the running dynamics and its choice can lead to sizable changes in the bound state masses. We describe top partners by a dual fermionic field in the bulk. Including suitable higher dimension operators can ensure a top mass consistent with the standard model.
\end{abstract}

DOI: 10.1103/PhysRevLett.126.071602

Extensions of the AdS/CFT correspondence [1-3] to less symmetric gauge/gravity duals have proved powerful in describing strongly coupled gauge theories. Adding probe branes allows quarks in the fundamental representation of the gauge group to be introduced [4] and to study the related meson operators $[5,6]$. These methods were successfully used to obtain gravity duals of chiral symmetry breaking $(\chi \mathrm{SB})$ and pseudo-Goldstone bosons in confining non-Abelian gauge theories [7,8]. A natural extension is to apply this approach to other strongly coupled theories in the context of particle physics. Our attention here is on composite Higgs models (CHM) of beyond the standard model physics, as reviewed in $[9,10]$.

The key element of CHM is a strongly coupled gauge theory, like $\mathrm{QCD}$, causing $\chi \mathrm{SB}$ in the fermion sector and generating four or more Nambu-Goldstone bosons, or "pions" [11]. By weakly gauging the global chiral symmetries, four then pseudo-Nambu Goldstone bosons (PNGBs) can be placed in a doublet of $\mathrm{SU}(2)_{L}$ to become the complex Higgs field. The composite nature of the Higgs removes the huge level of fine tuning in the standard model (SM) hierarchy problem. This strong dynamics would occur at a scale of $1-5 \mathrm{TeV}$, the expected scale for bound states. The LHC has started and, in future runs, will continue to search for such states.

There is some lattice gauge theory work on CHMs [12-16]. It is limited, though, by the cost of numerics and

Published by the American Physical Society under the terms of the Creative Commons Attribution 4.0 International license. Further distribution of this work must maintain attribution to the author(s) and the published article's title, journal citation, and DOI. Funded by SCOAP ${ }^{3}$. the inability to unquench fields (i.e., to include the effect of flavor modes on the gauge fields) and to match the models' precise fermion content. Here, we use nonconformal gauge/gravity models that explicitly include the gauge theories' dynamics through the running of the anomalous dimension $\gamma$ of the fermion or "quark" mass in the CHM. Our models are inspired by top-down models involving probe D-branes embedded into ten-dimensional supergravity-we retain as much of the Dirac-Born-Infeld structure as we can in our models to correctly capture the true AdS/CFT dynamics. However, we combine this with a phenomenological approach and insert sensible guesses for the running of $\gamma$ based on perturbation theory. We then predict some of the mesonic and baryonic spectrum of the theory. These top-down inspired holographic models describe the running dynamics of the specific CHM rather than the more generic previous analyses, e.g., the RandallSundrum [17] approach of $[18,19]$.

We consider two models: an $\mathrm{Sp}(4)$ theory with four fundamental and six sextet Weyl quarks [20]; and an SU(4) theory with five sextet Weyl and three fundamental Dirac quarks [21,22]. Both models can incorporate a SM Higgs among their PNGBs. We present results for the mass spectrum of bound states analogous to mesons in these theories and compare to relevant lattice results: for the $\mathrm{Sp}$ (4) theory, lattice results were obtained for the quenched theory; for the SU(4) theory, they have a slightly different fermion content with an even number of multiplets. The pattern of masses is well reproduced by the holographic model, although precise values can differ by up to $20 \%$. This gives us confidence to trust our predictions for how the masses change as we unquench, including the effect of flavors on the running coupling, and move to the true fermion content of the models. In particular, unquenching 
tends to separate the scales of the sextet and fundamental matter mesons. Slowing the running also reduces the scalar meson masses [23].

Finally, we address the generation of the top quark's large mass in CHMs, while also keeping flavor changing neutral currents under control. This can be achieved by the mechanism of partial compositeness [24] to generate the top-Higgs Yukawa coupling with higher dimension operators (HDOs) from a flavor scale above the strong dynamics scale. These CHM use a mechanism where the left $t_{L}$ and right handed $t_{R}$ top quarks mix with baryonlike "top partner" spin $1 / 2$ states $T_{L}, T_{R}$ in the gauge theory with the same quantum numbers [24]. The top partners are involved in the strong dynamics and so have an order one Yukawa coupling to the Higgs. HDOs then mix the top and top-partner fields to generate the top Yukawa coupling. To achieve the large top mass though requires top partner masses of the order of $800 \mathrm{GeV}$, which is below the natural baryon mass scale and not consistent with current LHC data [25-27].

We address this issue by first including a spinor of appropriate AdS mass into the holographic model, dual to the top partner operator in the field theory. This allows us to calculate the top partners' mass. A novel technical ingredient in this respect is the inclusion of spinor fields into a nonsupersymmetric bulk theory, for which we adapt previous results for supersymmetric probe branes [29]. We also add appropriate strongly coupled HDOs to the holographic model using Witten's double trace prescription [30]. The particular HDO we pick reduces to a shift in the top partners' mass at low energies. We show that the top Yukawa coupling can be made of order one by lowering the top partners' mass to roughly half the CHM's vector meson mass. This is plausibly reconcilable with experimental constraints.

Dynamic AdS/YM. - Our holographic model [31] is based on the Dirac-Born-Infeld action of a top-down model with a D7-brane embedded in a (deformed) $\mathrm{AdS}_{5}$ geometry. The deformation is expanded to quadratic order in the embedding function $X$ (see [32,33]). We add an axial gauge field as in AdS/QCD models [34,35] and a spinor. The model describes either a single quark in the background of the gauge fields and other quarks; or, by placing the fields in the adjoint of flavor and tracing over the action, multiple mass-degenerate quarks. The vacuum $X$ function will cause a global symmetry breaking pattern $G \rightarrow H$.

The model has a dimension one field for each gauge invariant operator of dimension three in the field theory. $X=L e^{i \pi}$ is dual to the complex quark bilinear or any other suitable bilinear operator, depending on the group and representation considered. This field's fluctuations are dual to the analog of the scalar and pseudoscalar $\sigma$ and $\pi$ mesons of the theory. $A_{L}^{\mu}$ and $A_{R}^{\mu}$ are dual to the analogs of the vector and axial vector mesons $V$ and $A$.

The gravity action of dynamic AdS/YM is, including also a spinor field [36],

$$
\begin{aligned}
S= & \int d^{5} x \rho^{3}\left\{\frac{1}{r^{2}}|D X|^{2}+\frac{\Delta m^{2}}{\rho^{2}}|X|^{2}\right. \\
& +\frac{1}{2 g_{5}^{2}}\left[F_{L, M N} F_{L}^{M N}+(L \leftrightarrow R)\right] \\
& \left.+\bar{\Psi}\left(\not D_{\mathrm{AAdS}}-m\right) \Psi\right\} .
\end{aligned}
$$

The five-dimensional coupling is obtained by matching to the UV vector-vector correlator, $g_{5}^{2}=\left\{48 \pi^{2} /\left[d(R) N_{f}(R)\right]\right\}$ [34]. $d(R)$ is the dimension of the quark's representation and $N_{f}(R)$ is the number of Weyl flavors in that representation. The model lives in a five-dimensional asymptotically AdS (AAdS) spacetime

$$
d s^{2}=r^{2} d x_{(1,3)}^{2}+\frac{d \rho^{2}}{r^{2}},
$$

with $r^{2}=\rho^{2}+L^{2}$ the holographic radial direction, corresponding to the energy scale, and $d x_{(1,3)}^{2}$ a four-dimensional Minkowski spacetime. The $\rho$ and $L$ factors in the action and metric are implemented directly from the top-down analysis of the $D 3 /$ probe- $D 7$ system. They ensure an appropriate UV behavior. In the IR, the fluctuations know about any $\chi \mathrm{SB}$ through $L \neq 0$.

The dynamics of a particular gauge theory, including quark contributions to any running coupling, is included through $\Delta m^{2}$ in Eq. (1). To find the theory's vacuum, with a nonzero chiral condensate, we set all fields to zero except for $L(\rho)$. For $\Delta m^{2}$ a constant, the equation of motion obtained from Eq. (1) is

$$
\partial_{\rho}\left(\rho^{3} \partial_{\rho} L\right)-\rho \Delta m^{2} L=0 .
$$

The solution takes the form $L(\rho)=m \rho^{-\gamma}+c \rho^{\gamma-2}$, with $\Delta m^{2}=\gamma(\gamma-2)$ in units of the inverse AdS radius squared. Here, $\gamma$ is the anomalous dimension of the quark mass. The Breitenlohner-Freedman bound [37], below which an instability to $\chi \mathrm{SB}$ occurs, is given by $\Delta m^{2}=-1$.

We impose a particular gauge theory's dynamics by using its running $\gamma$ to determine $\Delta m^{2}$ - as usual in holography, $M^{2}=\Delta(\Delta-4)$, with $\Delta$ the operator scaling dimension. For $\gamma<1$, we find $\Delta m^{2}=-2 \gamma$, thus a theory triggers $\chi \mathrm{SB}$ if $\gamma$ passes through $1 / 2$. Since the true running of $\gamma$ is not known nonperturbatively, we extend the perturbative results as a function of renormalization group scale to the nonperturbative regime. To find the running of $\gamma$, we use the perturbative results. To provide some understanding of error range in the holographic model, we compute with both the one-loop result $\gamma_{1 l}=\left\{\left[3 C_{2}(R)\right] / 2 \pi\right\} \alpha$ and the two-loop result $\gamma_{2 l}=\left(\alpha / 2 \pi^{2}\right)\left[\frac{3}{2} C_{2}(R)^{2}+\frac{97}{6} C_{2}(R) C_{2}(G)-\right.$ $\left.\left(10 N_{f} / 3\right) C_{2}(R) T(R)\right]$ with a running $\alpha$ [38]. Note that we decouple fields from the running as they go on mass shell. We average the results and use half the range as the error. 
We numerically solve (3) with our ansatz for $\Delta m^{2}$, using the IR boundary conditions $L\left(\rho=\rho_{\mathrm{IR}}\right)=\rho_{\mathrm{IR}}$, $\partial_{\rho} L\left(\rho=\rho_{\mathrm{IR}}\right)=0$. They are imported from the $D 3 / D 7$ system but imposed at the scale where the quarks go on mass shell. The spectrum is determined by considering fluctuations in all fields of (1) about the vacuum [31] see Supplemental Material [40] for details.

We will use this model to explore two gauge theories that have been proposed to underlie CHM-we pick the two with the most extensive lattice data. We denote models by their gauge group and the number of Weyl flavors in the fundamental $(F)$, and two-index antisymmetric $\left(A_{2}\right)$ representations.

Sp(4) gauge theory-Sp(4)4F, 6A2.-This CHM, proposed in [20], has top partners. Lattice studies related to this model were performed in $[12,13]$. The fundamental of $\mathrm{Sp}(2 N)$ is pseudoreal, hence the $\mathrm{SU}(2)_{L} \times \mathrm{SU}(2)_{R}$ symmetry of a model with four Weyl quarks in the fundamental representation is enhanced to an SU(4) flavor symmetry [41]. The condensation pattern is the same as in QCD, and breaks the $G=\mathrm{SU}(4)$ flavor symmetry to $H=$ $\mathrm{Sp}(4)$ with 5 PNGBs.

The top partners are introduced by including three additional Dirac fermions in the $A_{2}$ representation of the gauge group for $N>1$. It is natural to concentrate on the minimal, $\mathrm{Sp}(4)$, gauge group case. The top partners are $F A_{2} F$ bound states. From the point of view of the $\mathrm{Sp}(4)$ dynamics, there is an $G=\mathrm{SU}(6)$ symmetry on the six $A_{2}$ Weyl fermions that are in a real representation. The $A_{2}$ condensate breaks this symmetry to $H=\mathrm{SO}(6)$.

The $A_{2}$ fermions condense ahead of the fundamental fields, since the critical value for $\alpha$ where $\gamma=1 / 2$ is smaller (at the level of the approximations we use the critical couplings are $\alpha_{c}^{A_{2}}=\pi / 6=0.53, \quad \alpha_{c}^{F}=$ $4 \pi / 15=0.84)$.

We perform the AdS/YM analysis for the two fermion sectors separately, although they are linked since both flavors contribute to the running of $\gamma$ down to their IR mass scale. We find the $L(\rho)$ functions for the $A_{2}$ and $F$ sectors. We fluctuate around each embedding separately to find the spectrum (neglecting any mixing). We present the holographically computed spectrum in Table I. The errors show the range in using $\gamma_{1 l}$ or $\gamma_{2 l}$ and are mostly small. The scalar masses are dependent on the strength of the running and so have the largest errors.

Lattice data exist for the quenched theory [12]. To compare, we provide holographic results for the quenched case-here no fermions contribute to the running. The $V$ meson masses fit the lattice data well. For the $A$ mesons, the holographic model predicts close to degeneracy between the $F$ and $A_{2}$ sectors, while the lattice has a wider spread (although with large errors on the spread) and a 20\% higher estimate of the scale. The holographic pion decay constants are low by $20 \%-30 \%$. The lattice results are for the $a_{0}$ masses, not for the $\sigma$, but we include these data points for
TABLE I. AdS $/ \operatorname{Sp}(4) 4 F, 6 A_{2}$. Ground state masses for the vector, axial-vector, and scalar mesons for the two representations, $F$ and $A_{2}$, from holography (errors show the range in using $\gamma_{1 l}$ and $\left.\gamma_{2 l}\right)$ and the lattice. Also the pion decay constant and two estimates of the top partner baryon mass. We have rescaled the lattice data to the $M_{V A_{2}}$ mass in the quenched case [42] and for the unquenched case, where no $A_{2}$ states are included, rescaled so $M_{V F}$ matches the quenched case for comparison.

\begin{tabular}{lcccc}
\hline \hline & $\operatorname{AdS} / \mathrm{Sp}(4)$ & $\operatorname{AdS} / \mathrm{Sp}(4)$ & Lattice [12] & Lattice [13] \\
\hline & unquench & quench & quench & unquench \\
\hline$f_{\pi A_{2}}$ & $0.118(02)$ & $0.104(02)$ & $0.1453(12)$ & \\
$f_{\pi F}$ & $0.068(02)$ & $0.074(02)$ & $0.1079(52)$ & $0.1018(83)$ \\
$M_{V A_{2}}$ & $1^{*}$ & $1^{*}$ & $1.000(32)$ & \\
$M_{V F}$ & $0.783(31)$ & $0.92(04)$ & $0.83(19)$ & $0.83(27)$ \\
$M_{A A_{2}}$ & $1.35(01)$ & $1.29(02)$ & $1.75(13)$ & \\
$M_{A F}$ & $1.16(03)$ & $1.32(04)$ & $1.32(18)$ & $1.34(14)$ \\
$M_{S A_{2}}$ & $0.37(01)$ & $0.98(16)$ & $1.65(15)^{\text {a }}$ & \\
$M_{S F}$ & $0.77(13)$ & $1.10(15)$ & $1.52(11)^{\text {a }}$ & $1.40(19)$ \\
$M_{T A_{2}}$ & $1.85(01)$ & $1.85(05)$ & & \\
$M_{T F}$ & $1.46(07)$ & $1.71(08)$ & & \\
\hline \hline
\end{tabular}

${ }^{a}$ Note the lattice scalar results are for the $a_{0}$, not for the $\sigma$ we compute in the holographic model.

comparison. Overall, the success in the pattern suffices to make us trust changes as the theory is unquenched. Particularly, the gap between quantities in the $A_{2}$ and $F$ sectors grow by $10 \%-20 \%$ as the running between the condensation scales slows due to the $A_{2}$ being integrated out, and the scalar masses fall as the running slows. There are unquenched lattice results but only for the $F$ sector [13], so they do not shed light on the mass gap between the sectors or the dependence of the $\sigma$ mass.

$\mathrm{SU}(4)$ gauge theory-SU(4) $3 F, 3 \bar{F}, 5 A_{2}$.-We report on a second model $[21,22]$, for which there has also been related lattice work. The gauge group is SU(4). There are five Weyl fields in the $A_{2}$ representation. When these $A_{2}$ condense they break their $G=\mathrm{SU}(5)$ symmetry to $H=\mathrm{SO}(5)$ - the PNGBs include the Higgs. To include top partner baryons, fermions in the fundamental are added, allowing $\mathrm{FA}_{2} F$ states. The number of flavors is fixed to be three Dirac spinors, since we need to be able to weakly gauge the flavor symmetry to become the SU(3) of QCD. When these fields condense, the chiral $G=\mathrm{SU}(3)_{L} \times$ $\mathrm{SU}(3)_{R}$ symmetry is broken to the vector $H=\mathrm{SU}(3)$ subgroup. Note that this model is hard to simulate on the lattice due to the fermion doubling problem, so instead lattice work [14,15] has focused on the case with just two Dirac $A_{2} \mathrm{~S}$ and two fundamentals, which allows consideration of the unquenched case.

We display our holographic results in (II) with lattice data from [14]. The holographic approach and the lattice agree in the $V$ sector and the pion decay constants lie close to or just below the lower lattice error bar. We obtain additional masses that have not been computed on the lattice. Crucially, we move to the $5 A_{2}, 3 F, 3 \bar{F}$ fermion 
content by adding in the additional fields needed for the CHM of $[21,22]$. We see that the extra fermions slow the running between the $A_{2}$ and $F$ mass scales, reducing the $F$ sector masses by $10 \%$. The scalar masses again fall (by $20 \%$ ) in response to the slower running.

Top partners. - Top partners in both models we consider are $F A_{2} F$ states, spin-1/2 baryons of the strong dynamics. We describe them by a spinor fluctuating in AdS [43], dual to the baryon operator. From (1), we derive a second order wave equation for the spinor, given in [44], based on [29]. The main features are the following: states with $\Delta=9 / 2$, appropriate for three-quark states, have an AdS mass $m=5 / 2$. The spinor can be written as eigenstates of the $\gamma_{\rho}$ projector, with $\gamma_{\rho} \psi_{ \pm}= \pm \psi_{ \pm}$. The equation then splits into two copies of the dynamics (for $\psi_{+}$and for $\psi_{-}$) with explicit relations between the solutions [29]. One can solve one equation and from the UV behavior extract the source $\mathcal{J}$ and operator $\mathcal{O}$ values. See Supplemental Material [40] for further details.

This allows the baryon masses' computation for threefermion states associated with a particular background $L(\rho)$. Here, we wish to set $\mathcal{O}=F A_{2} F$. However, since it is a mixed state in principle the computation should take into account that the $A_{2}$ and $F$ states have distinct $L(\rho)$. We simplify this by using one $L(\rho)$, either that for $F$ or $A_{2}$, which assumes the flavors are degenerate-the two choices give predictions for the baryon masses, $M_{T A_{2}}$ and $M_{T F}$ at the bottom of Tables I and II. We expect the mass to lie between these two computations. There is only lattice data for the SU(4) model for the top partner-our estimate is $25 \%$ high, but sets a sensible figure from which to observe changes as we include HDOs.

The top mass itself is generated in CHM by the diagram in Fig. 1. The $Z$ factors are structure functions that depend on the strong dynamics. $g^{2} / \Lambda^{2}$ and the tilded factors on the second vertex in Fig. 1, which we will not distinguish henceforth, are the dimensionful couplings of the HDOs that mix the top and top partners of the form $\bar{t}_{L / R} \mathcal{O}$.

TABLE II. SU(4) theories-the spectrum in two scenarios and lattice data for comparison.

\begin{tabular}{lccc}
\hline \hline & Lattice SU(4) $[14]$ & AdS/SU(4) & AdS/SU(4) \\
\hline & $4 A_{2}, 2 F, 2 \bar{F}$ & $4 A_{2}, 2 F, 2 \bar{F}$ & $5 A_{2}, 3 F, 3 \bar{F}$ \\
\hline$f_{\pi A_{2}}$ & $0.15(4)$ & $0.105(06)$ & $0.111(01)$ \\
$f_{\pi F}$ & $0.11(2)$ & $0.094(01)$ & $0.108(01)$ \\
$M_{V A_{2}}$ & $1.00(4)$ & $1^{*}$ & $1^{*}$ \\
$M_{V F}$ & $0.93(7)$ & $0.91(03)$ & $0.88(02)$ \\
$M_{A A_{2}}$ & & $1.38(01)$ & $1.33(01)$ \\
$M_{A F}$ & & $1.35(02)$ & $1.21(02)$ \\
$M_{S A_{2}}$ & & $0.746(13)$ & $0.61(08)$ \\
$M_{S F}$ & & $0.829(19)$ & $0.66(01)$ \\
$M_{T A_{2}}$ & $1.4(1)$ & $1.85(01)$ & $1.84(01)$ \\
$M_{T F}$ & $1.4(1)$ & $1.69(06)$ & $1.63(05)$ \\
\hline \hline
\end{tabular}

On dimensional grounds, a sensible holographic estimate for the $Z_{3}$ factor is a weighted integral over the PNGB and baryon wave functions [45],

$$
Z_{3} \simeq \int d \rho \rho^{3} \frac{\partial_{\rho} \pi \psi_{B}^{2}}{\left(\rho^{2}+L^{2}\right)^{2}}
$$

A similar contributing term to the $Z$ and $\tilde{Z}$ factors is

$$
Z \simeq \tilde{Z} \simeq \int d \rho \rho^{3} \partial_{\rho} \psi_{B}
$$

$\pi$ and $\psi_{B}$ are $\rho$ dependent holographic wave functions for the PNGB and baryon, respectively. The $Z$ factor was computed on the lattice in [16] - for QCD it is expected that $Z \simeq 19 f_{\pi}^{3}$ and we find $Z=22.6 f_{\pi}^{3}$. For the SU(4) lattice variant, $Z \simeq 7.5 f_{\pi}^{3}$ is found, and we find $Z=7.0 f_{\pi}^{3}$ showing that our estimate is sensible.

We have then computed $y_{t}$ from the full set of factors in Fig. 1 in both $\mathrm{CH}$ models. If we set a cutoff for the HDOs roughly 6 times the vector meson mass, we find the top Yukawa coupling is only of order 0.01 , far below the needed value of 1 . This is the standard problem when trying to generate the top mass-it is suppressed both by the HDO scale $\Lambda$ and top partner mass squared.

Our new solution to this is to enhance $y_{t}$ by including a further HDO given by

$$
\mathcal{L}_{\mathrm{HDO}}=\frac{g_{T}^{2}}{\Lambda^{5}}\left|F A_{2} F\right|^{2} .
$$

As the operator $F A_{2} F$ becomes the top partner, this is directly a shift in its mass. To include this holographically, we use Witten's double trace prescription [30], according to which the vacuum expectation value $\left\langle F A_{2} F\right\rangle$ contributes to the source via $\mathcal{J}=\left(g_{T}^{2} / \Lambda^{5}\right)\left\langle F A_{2} F\right\rangle$ once (6) is turned on. From the asymptotic boundary behavior of the gravity solutions, we read off $\mathcal{J}$ and $\mathcal{O}$ and then compute $g_{T}$. From these results we can find the masses of the top partner for a particular $g_{T}$

The HDO (6) can indeed be used to reduce the top partner's mass as was shown in a more formal setting [29] -for small $g_{T}$ the effect is linear and small, but after a critical value the effect is much larger. The same conclusion

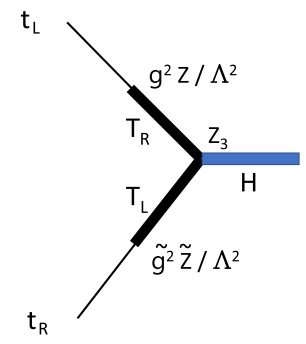

$$
y_{t}=\frac{g^{2} z \tilde{g}^{2} \tilde{z} z_{3}}{M_{T}^{2} \Lambda^{4}}
$$

FIG. 1. The top Yukawa coupling $\left(y_{t}\right)$ vertex diagram. 


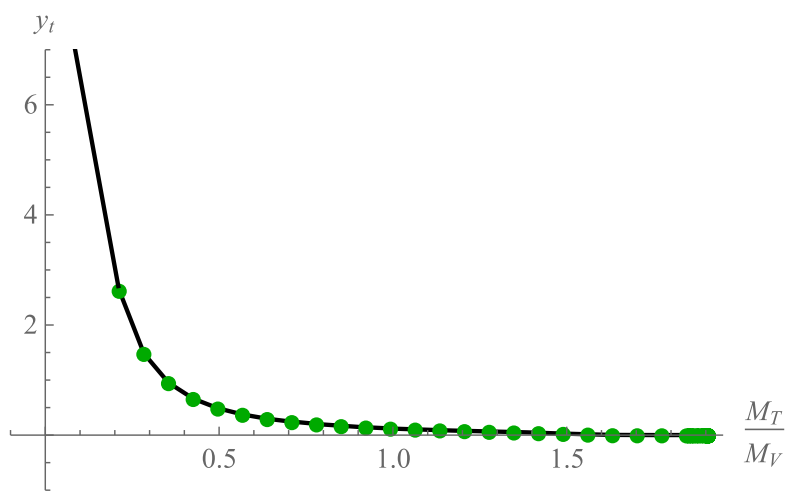

FIG. 2. Yukawa coupling as function of the top partner mass in the $A_{2}$ sector of the $\mathrm{SU}(4)$ gauge theory (here $g=\tilde{g}=1$ ).

was reached in a simple bottom-up model for the scalar mode as well [46]. In the two models above, using either of the baryon mass' estimates, one can reduce the top mass to half the $V$ meson mass (which is likely $0.5 \mathrm{TeV}$ or above in CHM) for $7<g_{T}<10$.

Caution is needed in computing the top Yukawa. As the top partner's mass changes, so do the $Z$ factors in (4) and (5). In particular, the HDO in (6) plays a large role since it induces a sizable nonnormalizable piece in the UV holographic wave function of the top partner. The integrals in the normalization factors for the state, which enter directly in the expressions for the $Z$ factors, are more dominated by the UV part of the integral. The overlap between different states can also change substantially. We therefore plot an example of the full expression for the Yukawa coupling from Fig. 1 against the top partner mass (which changes with $g_{T}$ ) in Fig. 2. We see that the top Yukawa grows as the top partner's mass falls and can become of order 1 as the top partners mass falls to about half of the vector meson mass. This suggests one might be able to realize a phenomenological viable top partner mass of $1 \mathrm{TeV}$ or so and the required top mass.

To conclude, we have shown that gauge/gravity duality methods are a powerful tool for obtaining sensible estimates in strongly coupled theories relevant to $\mathrm{CHM}$, and are thus a resource for model builders. The AdS/YM theory presented is fast to compute with. The fermion content can be changed in a simple way to obtain further models. Our results for CHM (and a much wider set of models that can be found in [44]) suggest how the spectrum will change as lattice simulations are unquenched to the correct flavor content and HDOs introduced.

We thank Raimond Abt, Alexander Broll, Tony Gherghetta, and Yang Liu for discussions. N.E. was supported by the STFC Grant No. ST/P000711/1.

*erdmenger@physik.uni-wuerzburg.de

†n.j.evans@soton.ac.uk porod@physik.uni-wuerzburg.de

k.c.rigatos@soton.ac.uk

[1] J. M. Maldacena, Int. J. Theor. Phys. 38, 1113 (1999).

[2] E. Witten, Adv. Theor. Math. Phys. 2, 253 (1998).

[3] S. Gubser, I. R. Klebanov, and A. M. Polyakov, Phys. Lett. B 428, 105 (1998).

[4] A. Karch and E. Katz, J. High Energy Phys. 06 (2002) 043.

[5] M. Kruczenski, D. Mateos, R. C. Myers, and D. J. Winters, J. High Energy Phys. 07 (2003) 049.

[6] J. Erdmenger, N. Evans, I. Kirsch, and E. Threlfall, Eur. Phys. J. A 35, 81 (2008).

[7] J. Babington, J. Erdmenger, N. J. Evans, Z. Guralnik, and I. Kirsch, Phys. Rev. D 69, 066007 (2004).

[8] M. Kruczenski, D. Mateos, R. C. Myers, and D. J. Winters, J. High Energy Phys. 05 (2004) 041.

[9] G. Cacciapaglia, C. Pica, and F. Sannino, Phys. Rep. 877, 1 (2020).

[10] G. Panico and A. Wulzer, The Composite Nambu-Goldstone Higgs (Springer, New York, 2016), Vol. 913.

[11] N. Arkani-Hamed, A. Cohen, E. Katz, and A. Nelson, J. High Energy Phys. 07 (2002) 034.

[12] E. Bennett, D. K. Hong, J.-W. Lee, C.-J. D. Lin, B. Lucini, M. Mesiti, M. Piai, J. Rantaharju, and D. Vadacchino, Phys. Rev. D 101, 074516 (2020).

[13] E. Bennett, D. K. Hong, J.-W. Lee, C.-J. D. Lin, B. Lucini, M. Piai, and D. Vadacchino, J. High Energy Phys. 12 (2019) 053.

[14] V. Ayyar, T. DeGrand, D. C. Hackett, W. I. Jay, E. T. Neil, Y. Shamir, and B. Svetitsky, Phys. Rev. D 97, 114505 (2018).

[15] V. Ayyar, T. DeGrand, M. Golterman, D. C. Hackett, W. I. Jay, E. T. Neil, Y. Shamir, and B. Svetitsky, Phys. Rev. D 97, 074505 (2018).

[16] V. Ayyar, T. DeGrand, D. C. Hackett, W. I. Jay, E. T. Neil, Y. Shamir, and B. Svetitsky, Phys. Rev. D 99, 094502 (2019).

[17] L. Randall and R. Sundrum, Phys. Rev. Lett. 83, 3370 (1999).

[18] R. Contino, Y. Nomura, and A. Pomarol, Nucl. Phys. B671, 148 (2003).

[19] K. Agashe, R. Contino, and A. Pomarol, Nucl. Phys. B719, 165 (2005).

[20] J. Barnard, T. Gherghetta, and T. S. Ray, J. High Energy Phys. 02 (2014) 002.

[21] G. Ferretti, J. High Energy Phys. 06 (2014) 142.

[22] G. Ferretti, J. High Energy Phys. 06 (2016) 107.

[23] N. Evans and K. Tuominen, Phys. Rev. D 87, 086003 (2013).

[24] D. B. Kaplan, Nucl. Phys. B365, 259 (1991).

[25] M. Aaboud et al. (ATLAS Collaboration), Phys. Rev. Lett. 121, 211801 (2018).

[26] A. M. Sirunyan et al. (CMS Collaboration), Phys. Rev. D 100, 072001 (2019).

[27] However, these bounds can be lower if nonstandard decays are important [28].

[28] G. Cacciapaglia, T. Flacke, M. Park, and M. Zhang, Phys. Lett. B 798, 135015 (2019).

[29] R. Abt, J. Erdmenger, N. Evans, and K. S. Rigatos, J. High Energy Phys. 11 (2019) 160.

[30] E. Witten, arXiv:hep-th/0112258.

[31] T. Alho, N. Evans, and K. Tuominen, Phys. Rev. D 88, 105016 (2013). 
[32] R. Alvares, N. Evans, and K.-Y. Kim, Phys. Rev. D 86, 026008 (2012).

[33] J. Erdmenger, N. Evans, and M. Scott, Phys. Rev. D 91, 085004 (2015).

[34] J. Erlich, E. Katz, D. T. Son, and M. A. Stephanov, Phys. Rev. Lett. 95, 261602 (2005).

[35] L. Da Rold and A. Pomarol, Nucl. Phys. B721, 79 (2005).

[36] $\not D_{\text {AAdS }}$ is the Dirac operator evaluated on Eq. (2).

[37] P. Breitenlohner and D. Z. Freedman, Ann. Phys. (N.Y.) 144, 249 (1982).

[38] For all the group theory factors we have used the Mathematica application LieART [39].

[39] R. Feger and T. W. Kephart, Comput. Phys. Commun. 192, 166 (2015).
[40] See Supplemental Material at http://link.aps.org/supplemental/ 10.1103/PhysRevLett.126.071602 for a brief exposition on the basics of fluctuations in the gravity model.

[41] R. Lewis, C. Pica, and F. Sannino, Phys. Rev. D 85, 014504 (2012).

[42] For a variable $C=(A / B)^{1 / 2}$ we translate errors as $d C=1 / 2\left[d A /(A B)^{1 / 2}+A^{1 / 2} d B / B^{3 / 2}\right]$.

[43] I. Kirsch, J. High Energy Phys. 09 (2006) 052.

[44] J. Erdmenger, N. Evans, W. Porod, and K. S. Rigatos, arXiv:2010.10279.

[45] To compute the $Z_{3}$ factor, we would need a cubic term in (1). However, its coupling is not determined in the bottom-up approach. We use the estimate given in (4).

[46] Y. Buyukdag, Phys. Rev. D 102, 106018 (2020). 